\title{
NUEVAS BÚSQUEDAS EN LOS ESTUDIOS DE AUDIENCIAS TELEVISIVAS
}

MARIA REBECA PADILLA DE LA TORRE Departamento de Comunicación/UAA

Sonia Livingstone, Making sense of television. The psychology of audience interpretation. Segunda edición, London and New York, Routledge, 1998, 212 pp.

Sonia Livingstone es profesora de psicología social del London School of Economics and Political Science. Dirige el MSc en Media and Communications. Ha investigado y publicado sobre las audiencias televisivas. Sus más recientes publicaciones son Talk on Televisión (1994) y Mass Consumption and Personal Identity (1992). Actualmente trabaja en la obra Children, Young People and the Changing Enviroment, en la que se pregunta si las nuevas modalidades en los medios modifican las relaciones tradicionales entre las personas (audiencias) y los medios masivos.

En su libro Making sense of televisión, Livingstone explora las vetas de los debates que han perfilado las búsquedas y el desarrollo de los estudios de audiencias y propone un nuevo camino: la psicología social. Su contribución consiste en recuperar la tradición de los estudios sociológicos y psicológicos de la comunicación masiva e integrarlos con los aportes de los estudios culturales y la crítica literaria para avanzar en la investigación de la interpretación, no sólo de los textos televisivos, sino en general, en la comprensión de cómo el individuo interpreta su mundo.

Analiza detalladamente las principales teorías cognitivas de la psicología social, las premisas de la au- 
diencia activa, la semiótica y teoría de la narrativa para diseñar tres investigaciones empíricas. Propone nuevos métodos para explorar empíricamente los procesos del televidente. Las preguntas que se plantean en esta obra son: ¿Cómo las personas producen sentido, cómo las representaciones de los medios se relacionan con o se incorporan en el conocimiento de la vida cotidiana, cómo diferentes personas interpretan la televisión de diferentes maneras y cómo la interpretación de las audiencias constituye la centralidad de las inquietudes en la investigación del campo en torno a la influencia y significado de los medios?

Esta autora toma como textos los programas televisivos, lo cual implica reconocer las interrelaciones complejas entre los diversos aspectos de los significados, las prácticas culturales de las cuales dependen, los requerimientos y competencias que el texto exige para que la persona lo comprenda. Desplaza las nociones de estŕmulo externo y de la persona como un ser sólo capaz de responder, pasivo y sin poder; por la metáfora del texto con su correspondiente lector o lectores.

La historia de la psicología social y de los estudios de la comunicación masiva tienen varios aspectos en común, que nos relata Sonia Livingstone, entre ellos su oscilación entre la pasividad del individuo y el reconocimiento de su poder. Ambas posiciones se han interesado por la construcción activa de los significados culturales, y los estudios de efectos, los experimentales y los conductistas han sido desplazados por una comprensión más compleja del análisis de la construcción social de la realidad. Sin embargo, la autora aclara que en principio la pregunta central de los efectos en torno a la influencia de los medios en nuestras vidas permanece vigente, $\mathrm{y}$ ahora no se discute el poder o no de las audiencias, sino las condiciones en las cuales se relativiza ese poder frente a la televisión para negociar la construcción social de los significados.

La postura de la audiencia activa ha sido duramente criticada por sobredimensionar su aspecto constructivista, negando el papel del texto o del referente mediático, sea la televisión u otro medio. También se le ha acusado de ser sobrecognitiva, minimizando el papel de las emociones y las acciones; e individualista, reduciendo el acto social de la televidencia al acto cognitivo individual. Livingstone propone no dejar a 
un lado estos aspectos, sino recuperar el valor del texto y de los contextos del televidente como elementos que limitan y condicionan la producción de sentidos.

Livingstone decide aplicar los aportes de la psicología social al campo de los estudios de recepción televisiva en estudios empíricos concretos, y para ello elige a las soap operas (telenovelas) cómo el género/ texto televisivo que analizará. Argumenta que las características de este género televisivo constituyen un marco en donde se puede dar un seguimiento más claro de los procesos del televidente en la comprensión de las convenciones del género, sus motivaciones para ser sus espectadores continuos y sus recursos sociocognitivos -para producir sentidos en referencia a sus personajes y narrativa.

La propuesta de Livingstone busca analizar la convergencia entre los elementos del texto que permiten la comprensión psicológica por parte de sus televidentes, en un primer momento; para profundizar en sus posibles interpretaciones y los usos sociales de las soap operas en una segunda instancia, es en este último terreno en el cual la mayoría de los estudios de recepción han trabajado. La convergencia se da en el retomo a la identificación sistemática de ciertos patrones narrativos que propone el texto, para contrastarlos con la comprensión de la narrativa por parte de sus televidentes. Esta dimensión, nutrida por conceptos claves de la semiótica y la teoría literaria, sería el enlace entre el estudio de las posibilidades del texto y la indagación de la producción de significados en las audiencias.

La hipótesis de la autora se refiere a que existen fronteras en el texto en cuanto a que las narrativas de un género específico cuentan una relación de eventos que conducen la atención y la comprensión de la audiencia de una manera convencional y concreta, y que se puede medir el grado de correlación entre el texto y el lector, a su vez, existe otra dimensión que abre la obra a las interpretaciones de la audiencia, las cuales no pueden medirse o analizarse como correctas o incorrectas porque se refieren a los procesos de negociación de significados en donde se involucran más elementos que el texto y el lector.

¿Qué tanto es el texto un conjunto de instrucciones para que el lector las aplique? o ¿qué tanto es un conjunto de invitaciones o sugerencias, con preferencias más que directrices 
para el lector?

Eco comprende el papel del lector en términos de los códigos del texto, derivados del autor, el género y el contexto cultural de la producción y de los del lector, derivados de su experiencia y posición sociocultural de recepción. Existen lecturas aberrantes en donde no hay relación entre el texto y lector, y dependen de la apertura o cerrazón del texto, debido a que existen textos que buscan las posibilidades de la interpretación de manera intencionada.

En las tres soap operas estudiadas: Dallas, Coronation Street y East Enders, los sujetos pertenecían a diferentes edades y la mayoría eran trabajadores, empleados y algunos profesionistas. El requisito principal para elegir a los informantes fue el de ser televidentes regulares de las soap operas durante varios años; en el caso de la soap opera británica Coronation Street, algunos señalaron de haberla visto durante veinte años.

En un primer estudio, se les pidió a las personas agrupar a los personajes de acuerdo a su parecido y se representaron sus similitudes y oposiciones espacialmente mediante el método de escala multidimensional. El análisis empírico mostró que los temas o dimensiones dominantes fue- ron la moralidad, el poder; el género y la manera de orientarse en la vida, que la autora distingue como los personajes tradicionales, serios y rígidos de aquéllos más modernos, alegres y flexibles. ¿Qué tanto estas oposiciones son presentadas por el texto y qué tanto representan la construcción de un significado guiado por un conocimiento social común?

Livingstone encontró en estos resultados que los estereotipos presentes en el conocimiento social no fueron la estructura clave a partir de la cual los informantes representaron a los personajes, el papel del conocimiento social opera en parte frente a la estructura del programa. Los informantes cambiaron sus marcos estereotípicos de acuerdo a la estructura del programa.

Esta investigadora relaciona los tres ejes encontrados con mayor peso en la representación de los personajes: moralidad, poder y género; con los tres ejes que estructuran la percepción de las personas según Osgood de evaluación, potencia y género. De esta manera, la autora relaciona la representación de los personajes de las soap operas con la de las personas en la vida cotidiana.

Concluye que tanto el conocimiento social como la estructura del texto 
son determinantes en las representaciones de los televidentes y cada uno modifica o matiza la influencia del otro. La representación resultante depende de lo aportado por ambas y de la naturaleza de la negociación entre sí.

Livingstone argumenta que los estudios de recepción han subestimado el papel del texto y la dependencia del significado en torno a la interacción entre el texto y el lector. Atinadamente señala la falta de un mayor número de estudios empíricos frente al mero debate teórico, y a las especulaciones en la empresa por conocer a las audiencias contemporáneas. Caso que sucede con mucha frecuencia en países como México. También sostiene que los estudios de recepción no deben fundamentarse sólo en la diversidad. Como muestra su estudio, existe un alto grado de consenso en la lectura de las soap operas en un nivel general y las divergencias y variedad en la recepción se dan en niveles más específicos. Por ello diseña otra investigación para construir una taxonomía inicial de los tipos de divergencia.

En este segundo estudio, $27 \mathrm{mu}-$ jeres fueron detectadas como televidentes asiduas de Coronation Street y se les solicitó recordaran tres na- rrativas o historias dentro de esta soap opera, narrando los sucesos. Comparando las diversas interpretaciones, los hechos fueron registrados de manera similar, en donde la divergencia se manifestó fue en lo que los televidentes "hacen" con la información.

Los resultados fueron los siguientes: el estudio de las interpretaciones de las narrativas de los televidentes en condiciones naturales ilustra que los personajes y la narrativa están íntimamente relacionados porque los espectadores utilizan su conocimiento de los personajes para crear significados coherentes y consistentes de los eventos que perciben. Existe divergencia en la interpretación de sŭs televidencias en las siguientes modalidades: a) la evaluación de un personaje es generalmente positiva o negativa; b) la narrativa se centra en algún aspecto como la personalidad o el comportamiento; c) en la complejidad de la interpretación; y d) la perspectiva de un personaje que se elige para observar los sucesos. Se comprobó un mayor grado de consenso que de divergencia en la interpretación de los televidentes, el consenso se revela en los juicios hacia las personalidades de los personajes y en el predominio de ciertas interpreta- 
ciones.

Los espectadores llevaron a cabo sus propias inferencias del relato, para complementar sus interpretaciones, recurriendo al conocimiento más general, mítico y culturalmente consensuado. Las mujeres construyeron interpretaciones siempre coherentes incluso en los relatos que contenían errores respecto a la historia original

En una tercera investigación se indagó sobre las diversas interpretaciones que una historia o conflicto específico, dentro de la continuidad narrativa de una soap opera -Coronation Street-, puede generar. A 66 informantes se les solicitó contestaran tres clases de preguntas: sobre datos generales de su persona, la identificación con, el reconocimiento de, la evaluación de y la elección de partido con respecto a algunos de los personajes. La tercera pregunta se refería al grado de desacuerdo con 30 enunciados derivados de otros relatos, comentarios e inferencias de un pequeño grupo de televidentes sobre esa historia en particular.

Las personas entrevistadas adoptaron diversas posturas con respecto al comportamiento de los personajes en la narrativa, mostrando que la televisión es un "foro cultural" en don- de se exhiben diversas formas de responder ante situaciones de la vida. La elección de posturas con respecto a los personajes involucrados en el conflicto de la historia de parte de las personas en el estudio, tuvo relación con su edad y género, además de la manera de relacionarse con los personajes. Así, los televidentes difieren en sus interpretaciones en relación con la postura adoptada frente al conflicto mostrado, la evaluación o juicio de las acciones de los personajes, el conocimiento inferido de algunas acciones de los personajes y predicciones sobre eventos futuros posibles.

Mediante estos estudios, Livingstone realiza un aporte analítico entre comprensión e interpretación. Describe a la comprensión, de parte de las audiencias, como el proceso de decodificación del nivel denotativo de significado del texto y en un segundo momento, el nivel connotativo del significado textual es decodificado a través de procesos de implicación y asociación; por último, la respuesta del televidente a estos significados decodificados depende de las circunstancias específicas de su persona y entorno. La comprensión se refiere entonces, a que a pesar de la complejidad de los textos, éstos convergen sobre información específica, 
a partir de la cual ciertas lecturas pueden valorarse como correctas o incorrectas.

La interpretación se centra en las maneras en las cuales el texto involucra marcos o narrativas convencionales para crear conexiones con significados ideológicos o míticos. Las interpretaciones no pueden juzgarse por su asertividad, se valoran como un producto de la experiencia del lector, generadas a partir de un mayor o menor grado de normas asumidas, o más o menos creativas, críticas o de interés. Habría que reflexionar en torno a cómo esta autora revierte la secuencia de la comprensión hermenéutica de la interpretación a la comprensión, por la interpretación a partir de la comprensión. Quizás sea sólo un cambio en la terminología.

Visto desde esta perspectiva, tradicionalmente los psicólogos se han interesado en los procesos de comprensión y los comunicólogos en lo relacionado a la interpretación. A pesar de las distintas tradiciones, es válido iniciar la convergencia a partir de la concepción de Eco de las lecturas divergentes o aberrantes. Una pregunta legítima sería cuestionar, en primer lugar, si las audiencias han recibido la información de manera correcta, para después con- cebir al texto como una obra abierta y polisémica y a las audiencias como constructoras activas de sus interpretaciones.

Livingstone se planteó como objetivo mostrar mediante estos estudios empíricos, cómo las personas relacionan su conocimiento del mundo con el mundo de la televisión, sin embargo, finalmente, no aporta en el terreno del comprender cómo el mundo de la televisión se relaciona con el mundo cotidiano y real. Es valiosa su postura en cuanto a que el conocimiento del lector no puede darse sin una relación con el texto y viceversa, los textos no pueden comprenderse sin su relación con sus lectores, en el campo de los estudios de recepción televisiva.

Esta investigadora admite que la integración que propone de las posturas en el campo de los estudios de audiencias: la administrativa o de transmisión y la orientada por un modelo cultural de la recepción televisa, es difícil, dadas las condiciones de inmadurez, tanto de la teoría de la recepción de audiencias televisivas como de las metodologías para comprender a las audiencias. Además, la autora señala las diferencias de origen epistemológico, político y teórico entre estos modelos; sin embargo 
aboga por el conocimiento de ambos caminos, que en muchas ocasiones se ignoran entre sí.

¿QuÉ SIGUE EN LA INVESTIGACIÓN DE LAS AUDIENCIAS?

Sonia Livingstone manifiesta su interés concreto por la interpretación de los textos y aboga por una concreción en los temas que atañen a los estudios de las audiencias, los cuales se pierden en el amplio terreno del consumo y la apropiación de los medios en la vida cotidiana.

El trabajo teórico y metodológico, de más de veinte años, de los estudios de audiencias fundamentó la posibilidad de considerar una audiencia activa e interpretativa, y dio cuenta de ello empíricamente. Estos postulados han sido enfrentados y han logrado sostenerse con éxito, las audiencias obtuvieron mayor reconocimiento y valor tanto en los terrenos académicos como políticos. Ahora, para esta autora siguen otras preguntas y respuestas más complejas y específicas para garantizar el desarrollo del campo de estudio de las audiencias y no permitir su estancamiento.

La autora señala dos focos de interés para la investigación futura: las audiencias se estructuran mediante factores textuales, como serían el texto cerrado, la lectura preferente, las convenciones del género, los discursos naturalizados, las ideologías dominantes y las posiciones del sujeto. En segundo lugar, las audiencias se estructuran a través de factores psicológicos, como posiciones sociodemográficas, capital cultural, comunidad interpretativa, discursos del contexto, recursos sociocognitivos, identidades nacionales o fuerzas psicodinámicas. Ambas determinantes-textuales y sociales-deben comprenderse en relación con el texto y las oportunidades sociales para la apertura, la contradicción, la agencia, la polisemia, la ambigüedad y demás, dado que juegan un papel clave en el análisis del cambio social y en la producción y reproducción de los significados de la vida cotidiana. Esta obra es valiosa porque expone de manera, muy detallada, teórica y empíricamente la construcción de una argumentación para esbozar un nuevo camino en la investigación de las audiencias. En Latinoamérica ha predominado el debate teórico en torno a las audiencias televisivas y los estudios empíricos de recepción televisiva, inscritos en la tradición de los estudios culturales, se les ha cri- 
ticado por su tendencia psicológica e individualista y su alejamiento de una comprensión más social y crítica de la realidad. A la luz de este estudio, a los estudios de recepción televisiva en México les faltaría mucho para integrar la teoría y la experiencia psicológica para sostener que los estudios de recepción son psicologistas. Por otra parte, es importante atender al llamado de Livingstone en el sentido de que se debe hacer un esfuer- zo por trascender el carácter cualitativo y exploratorio que los ha caracterizado hacia pautas más concretas y específicas en la metodología. No es válido mantenerse en el amplio espectro de la diversidad, como resultados de investigación. Ahora, las preguntas empíricas deben orientarse a cómo, dónde y bajo qué circunstancias diferentes significados se producen. 
